\title{
The Nature of Potential Fluctuations in the MBE CdTe/CdMgTe Quantum Well in a Magnetic Field: Experiment and Theory
}

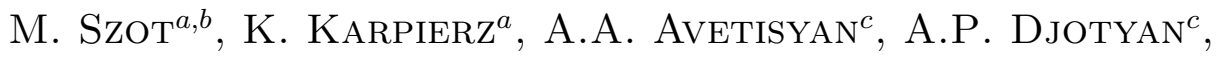 \\ J. Kossut ${ }^{b}$ And M. GRYNBerG ${ }^{a}$ \\ ${ }^{a}$ Institute of Experimental Physics, Warsaw University \\ Hoża 69, Warsaw, Poland \\ ${ }^{b}$ Institute of Physics, Polish Academy of Sciences \\ al. Lotników 32/46, Warsaw, Poland \\ ${ }^{c}$ Department of Physics, Yerevan State University, Yerevan, Armenia
}

Far infrared photoconductivity spectra of $\mathrm{CdTe} / \mathrm{Cd}_{0.8} \mathrm{Mg}_{0.2} \mathrm{Te}$ quantum well uniformly $n$-type doped with iodine in barriers and in the well were investigated as a function of the magnetic field. The spectra were recorded for several fixed far infrared photon energies and peaks corresponding to intra donor transitions were observed. The magnetic field at which the peaks were observed was the same for all far infrared photon energies used. This is interpreted as an evidence of the presence of fluctuations of the electrostatic potential - quantum dots which reduce the dimensionality of the potential in which a shallow donor is placed. A characteristic size of the fluctuations was found to be of $23-25 \mathrm{~nm}$ and $30-40 \mathrm{~nm}$. Theoretical calculations show a nonmonotonic dependence of the electron binding energy on the donor centre in such quantum dot, as a function of magnetic field. This explains why the position of experimentally observed peak is insensitive to far infrared photon energy used. Temperature evolution of spectra and the theoretical model proposed, indicate similarities between fluctuations in the two-dimensional structure investigated and fluctuations in bulk systems.

PACS numbers: 71.55.Gs, 72.15.Rn , 72.80.Ey, 73.21.Fg

\section{Introduction}

The problem of the electron energy in the vicinity of a Coulomb centre in a quantum well (QW) has been already theoretically solved for many years [1]. 
The results, confirmed by experiments $[1,2]$ show that — on the contrary to a $3 \mathrm{D}$ environment - in a 2D system the electron binding energy on a Coulomb centre depends on the centre location in the structure. The binding energy is the biggest in the middle of a QW and monotonically decreases when the donor is shifted towards the barrier. Experiments on $\delta$-doped samples (like GaAs/GaAlAs QW [3]), where the donor location in the structure is precisely defined, show that the application of the external magnetic field, $B$, leads to the Zeeman splitting of the donor states and enables us to observe intra donor transitions [3]. However, similar magnetospectroscopy experiments on QWs with donor positions distributed over all the structure show quite different results. Instead of sharp lines with positions corresponding to the Zeeman splitting energy, one observes only one, relatively broad line in the spectrum, whose position in the spectra is always the same, regardless far infrared (FIR) photon energy used [4]. The explanation of this surprising feature assumes a crucial role of local fluctuations of the electrostatic potential in localization of electrons onto donor centres, and was presented in previous papers $[4,5]$. The aim of the present work is to present a comparison of the investigated fluctuations with fluctuations of a 3D system, and to present a theoretical model which supports qualitative considerations explaining the experimental results up to now. The theoretical calculations are based on a variational approach of the Schrödinger equation in a quantum dot (QD) (a local fluctuation) in the presence of the Coulomb potential and the magnetic field.

\section{A model of a shallow donor in a quantum dot in the magnetic field}

Experiments done on $\mathrm{CdTe} / \mathrm{Cd}_{0.8} \mathrm{Mg}_{0.2} \mathrm{Te}$ quantum wells uniformly doped with iodine donors [4] showed that in this system one gets the conditions where a donor centre is "embedded" in a kind of a quantum dot, where the quantum confining potential is the following:

a) along the $z$ direction (the growth direction) - confinement due to the potential of the QW:

$$
V_{\mathrm{c}}^{\mathrm{qw}}(z)=\left\{\begin{array}{cc}
0, & |z| \leq d \\
V_{0}, & |z|>d
\end{array}\right.
$$

where $2 d$ - the width of quantum well along the $z$ direction, $V_{0}$ - depth of the QW,

b) in the $x y$ plane - due to the existence of local potential fluctuations originating from all ionized centres in the structure [4] and modeled by a parabolic potential $V_{\mathrm{c}}^{\mathrm{f}}$ :

$$
V_{\mathrm{c}}^{\mathrm{f}}(\rho)=\left\{\begin{array}{l}
\frac{1}{2} m^{*} \omega_{\mathrm{p}}^{2} \rho^{2}, \quad 0 \leq \rho \leq R_{\mathrm{f}}, \\
\frac{1}{2} m^{*} \omega_{\mathrm{p}}^{2} R_{\mathrm{f}}^{2}, \quad \rho>R_{\mathrm{f}},
\end{array}\right.
$$

where $\omega_{\mathrm{p}}$ is the frequency of oscillation in this potential, $\rho$ - the distance from the centre in the $x y$ plane, $R_{\mathrm{f}}$ - radius of the fluctuation describing its 
spatial extent in the $x y$ plane, $m^{*}$ — the electron effective mass. A schematic picture of the shape of the confining potential in the plane of the quantum well is presented in Fig. 1.

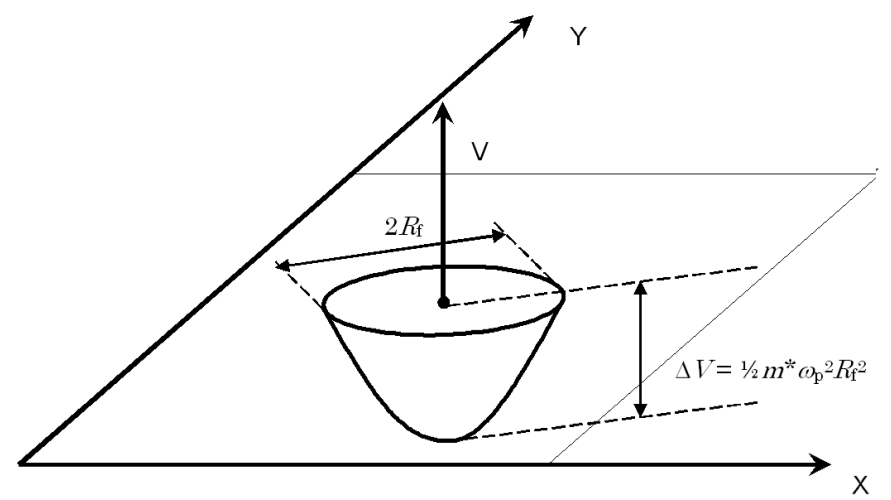

Fig. 1. The shape of the confining potential in the xy plane of the quantum well.

According to a qualitative model explaining the presence of one line at the same magnetic field [5], with increasing $B$, the magnetic length of a conduction band electron is decreasing and, for a certain value of $B^{*}$, becomes comparable to $R_{\mathrm{f}}$. This results in an enhanced electron localization in the fluctuation (in the quantum dot) and then on a shallow donor which is located within the extent of the fluctuation [5]. This localization enables us to observe intra-shallow donor transitions, which manifests as an increase of the magnetophotoconductivity signal. It is worth noticing that this increase is of a slightly different origin than that in a standard magnetospectroscopy experiment. A uniform doping of the sample assures that in the system investigated there is a variety of shallow donors located at each possible position with respect to the centre of the QW. This gives a continuum of the density of states (DOS) available for intra-donor transitions, and also a continuous range of energy of available intra-donor transitions: there is always a donor which can absorb a FIR photon sent onto the sample. An incident photon "chooses" a donor with the level splitting matching its energy. Thus the main reason of observing the intra-donor transition signal is the population of shallow donor states and not the transition energy matching.

\section{Experiment}

The samples investigated were single $\mathrm{CdTe} / \mathrm{Cd}_{0.8} \mathrm{Mg}_{0.2} \mathrm{Te}$ quantum wells, MBE grown on a semi insulating GaAs substrate and were described previously [5]. A $16 \mathrm{~nm}$ wide CdTe quantum well and $48 \mathrm{~nm}$ wide $\mathrm{Cd}_{0.8} \mathrm{Mg}_{0.2}$ Te barriers were uniformly $n$-doped by iodine at the level of $\sim 10^{16} \mathrm{~cm}^{-3}$. The quantum well was $240 \mathrm{meV}$ deep in the conduction band. On the top surface of the structure, indium contacts were attached allowing the in-plane flow of the current. 
Experiments were carried out by a standard magnetophotoconductivity technique with a molecular laser as the FIR source. A change of the sample conductivity due to absorption of the FIR light was measured. The photoconductivity (PC) spectra, measured as a function of the external magnetic field with a lock-in technique, were obtained at two temperatures: $T=1.5 \mathrm{~K}$ and $4.2 \mathrm{~K}$. During experiments the sample was additionally illuminated by visible light with the photon energy $h \nu=2.1 \mathrm{eV}$ (for experiments at $4.2 \mathrm{~K}$ ) or by red LED with the photon energy $\sim 1.9 \mathrm{eV}$ (for experiments at $1.5 \mathrm{~K}$ ). This illumination was necessary in order to decrease the sample resistance to the measurable level — less than $\sim 10 \mathrm{M} \Omega$.

Figure 2 shows the magnetophotoconductivity spectra obtained for the sample investigated at both temperatures and for two different energies in the case of the incident FIR photons. It is clearly seen that in all cases one observes a line covering a range from $\sim 1.5 \mathrm{~T}$ to $\sim 3-3.5 \mathrm{~T}$ for different photon energies. For lower temperature, there is also a second line, starting at $\sim 4.3 \mathrm{~T}$, which exhibits the same behaviour. For $T=4.2 \mathrm{~K}$, a clear observation of this peak is perturbed by the signal coming from the SI GaAs substrate. It is known that for illuminated SI GaAs, a nonequilibrium population of shallow donors can be seen by observation of intra shallow donor transitions [5]. This transition is also observed in

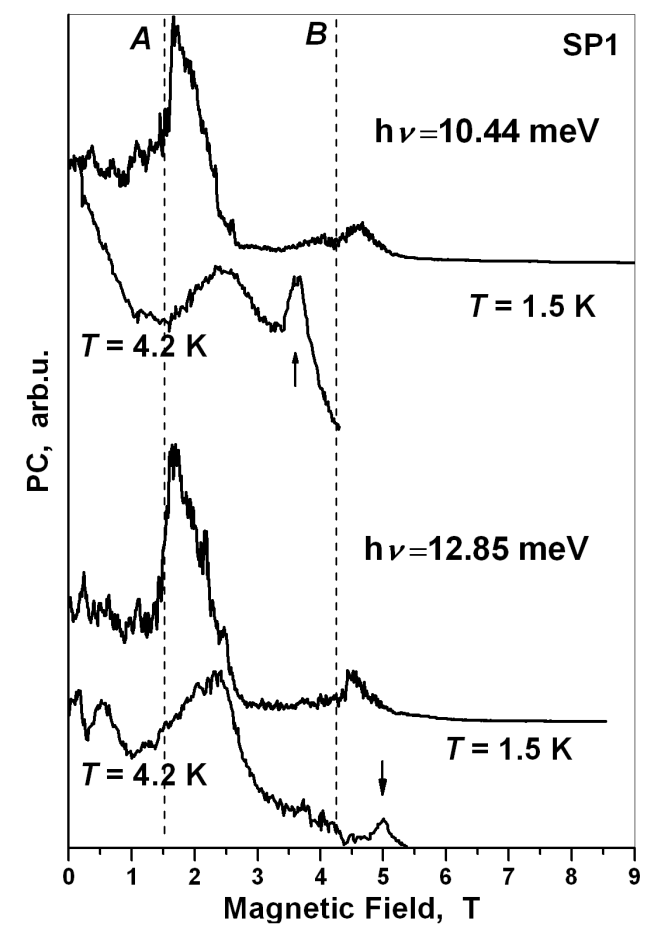

Fig. 2. Photoconductivity spectra for different FIR photon energies at two temperatures. The arrows mark positions of the peaks due to intra shallow donor transitions in SI GaAs substrate. The dashed lines correspond to the onset of lines observed. The spectra were shifted for better presentation. 
our sample and is marked by arrows in Fig. 2. This indicates that the visible and FIR light penetrate through the $\mathrm{CdTe} / \mathrm{CdMgTe}$ structure. An analysis shows that the magnetic field dependence of the energy of this transition follows the Zeeman splitting energy.

Assuming that the presence of lines $A$ and $B$ is governed by the magnetic field induced localization in the described above "quantum dots", and basing on the dependence of the cyclotron radius of conduction band electrons vs. the magnetic field: $r_{\mathrm{B}}=\sqrt{\hbar / e B}$, one can estimate the spatial extent $R_{\mathrm{f}}$ of a fluctuation in structure investigated. A rough approximation assuming that the localization occurs only when $R_{\mathrm{f}}$ is equal to the cyclotron radius gives the result that there are two kinds of quantum dots in the sample investigated: bigger ones with diameters of 30-40 nm (corresponding to the peak $A$ ) and smaller ones with the diameters in the range $23-25 \mathrm{~nm}$ (peak $B$ ).

\section{Interpretation}

Peak $B$ which corresponds to the smaller fluctuations is not visible at $4.2 \mathrm{~K}$. This allows one to conclude that the localization in smaller fluctuations is less probable at higher temperatures. One can propose that smaller quantum dots start to depopulate at higher temperatures and they are already empty at $4.2 \mathrm{~K}$. It means that either the parabolic confining potential is shallow enough to enable depopulation at $T=4.2 \mathrm{~K}(k T \approx 0.36 \mathrm{meV})$ or localization by the magnetic field is too weak and is vanishing at $4.2 \mathrm{~K}$. Thus the value of $\sim 0.3 \mathrm{meV}$ gives a rough estimate of the depth of the confining potential of smaller quantum dots. The larger dots, which are responsible for the peak $A$, do not depopulate at $4.2 \mathrm{~K}$. They are not only of larger dimensions but also significantly deeper. In that sense the observed fluctuations in our sample are similar to fluctuations in a $3 \mathrm{D}$ environment. In the latter case, a statistical distribution of ionized centres in a bulk, an infinite system, leads to potential fluctuations with the depth proportional to the spatial extent: $\Delta V \sim \sqrt{R_{\mathrm{f}}}[6]$. In the investigated $2 \mathrm{D}$ system, we also observe that larger fluctuations are deeper.

\section{Theoretical model}

The results of calculations presented below are the first step towards a quantitative understanding of the results experimentally obtained. They are based on solving the Schrödinger equation for a single electron bound by a Coulomb potential of a shallow donor in the presence of a confining potential of the quantum well and the potential of a quantum dot (i.e., a fluctuation) described in Sect. 2. The proposed Hamiltonian of an electron in the magnetic field (in SI system) has therefore the following form:

$$
\begin{aligned}
H= & \frac{1}{2 m^{*}}(\hat{\boldsymbol{p}}+e \boldsymbol{A})^{2}-\frac{e^{2}}{4 \pi \kappa \sqrt{\rho^{2}-\rho_{0}^{2}-2 \rho \rho_{0} \cos \varphi+\left(z-z_{0}\right)^{2}}} \\
& +V_{\mathrm{c}}^{\mathrm{f}}(\rho)+V_{\mathrm{c}}^{\mathrm{qw}}(z),
\end{aligned}
$$


where $m^{*}, \rho, V_{\mathrm{c}}^{\mathrm{f}}, V_{\mathrm{c}}^{\mathrm{qw}}$ - are the same as in Sect. 2, $\boldsymbol{A}$ - is the vector potential of the magnetic field $\boldsymbol{B}$, applied along $z$ direction, chosen as $\boldsymbol{A}(r)=$ $\frac{1}{2} \boldsymbol{B} \times \boldsymbol{r},\left(\rho_{0}, z_{0}, \varphi_{0}=0\right)$ are the donor centre coordinates in a cylindrical frame of reference and $\kappa$ is the static dielectric constant. We note that the origin of the coordination system is placed in the centre of the $\mathrm{QD}$, not on the shallow donor. The solution of the Schrödinger equation requires rewriting it in dimensionless units. This is done by introducing the atomic units of distance and energy - the Bohr radius - equal to $a_{\mathrm{B}}^{*}=4 \pi \hbar^{2} \kappa / m^{*} e^{2}$, and the effective Rydberg energy equal to $R y^{*}=m^{*} e^{4} / 32 \pi^{2} \kappa^{2} \hbar^{2}$, as well as a dimensionless measure of the magnetic field, $\gamma=\left(\frac{a_{\mathrm{B}}}{r_{\mathrm{B}}}\right)^{2}=\hbar \omega_{\mathrm{c}} / 2 R y^{*}$. The latter value can be also expressed as: $\gamma=16 \pi^{2} \hbar^{3} \kappa^{2} B / m^{* 2} e^{3}$. The dimensionless Coulomb potential is equal to: $U_{\text {Coul }}(\rho, z, \varphi)=-2 / \sqrt{\rho^{2}+\rho_{0}^{2}-2 \rho \rho_{0} \cos \varphi+\left(z-z_{0}\right)^{2}}$ as well as $V_{\mathrm{c}}^{\mathrm{f}}(\rho)=\gamma_{\mathrm{p}}^{2} \rho^{2}$ for $0 \leq \rho \leq R_{\mathrm{f}} / a_{\mathrm{B}}^{*}$, where $\gamma_{\mathrm{p}}=\hbar \omega_{\mathrm{p}} / 2 R y^{*}$. The dimensionless quantum well potential along the $z$ direction has the value of $V_{0} / R y^{*}$. In the above equations $\rho, \rho_{0}$, and $z$ are dimensionless measure of relevant positions.

We have chosen the ground state variational wave function of the system in a way that it takes into account the Coulomb interaction between the donor and the electron as well as all confinements in the system [7]:

$$
\psi=N \exp \left(-\lambda \sqrt{\rho^{2}+\rho_{0}^{2}-2 \rho \rho_{0} \cos (\varphi)+\left(z-z_{0}\right)^{2}}\right) \exp \left(-\gamma_{\mathrm{eff}} \rho^{2} / 2\right) f(z),
$$

where $N$ is a normalization constant, variational parameter $\lambda$ takes into account the Coulomb interaction, the factor $\exp \left(-\gamma_{\mathrm{eff}} \rho^{2} / 2\right)$ describes the influence of the fluctuation potential and the magnetic field strength: $\gamma_{\mathrm{eff}}=\sqrt{\gamma^{2}+4 \gamma_{\mathrm{p}}^{2}}, f(z)$ describes the influence of quantum well potential,

$$
f(z)= \begin{cases}\cos (\alpha z), & |z| \leq d \\ \cos (\alpha d) \exp (\beta d) \exp (\beta z), & z<-d \\ \cos (\alpha d) \exp (\beta d) \exp (-\beta z), & z>d\end{cases}
$$

where $\alpha^{2}=\varepsilon_{1}, \beta^{2}=V_{0}-\alpha^{2}$, and $\varepsilon_{1}$ is the lowest energy of a free electron in a quantum well in the absence of the Coulomb interaction, magnetic field and fluctuation, obtained from the equation $-\sqrt{V_{0}-\varepsilon} \tan d \sqrt{\varepsilon}=\sqrt{\varepsilon}$ (the energy of the bottom of the first Landau level in the quantum well). For ground state energy (1s state) of the system we obtain the analytical expression on the base of the equation $E_{\text {total }}=\langle\psi|H| \psi\rangle$. The binding energy $E_{\mathrm{B}}$ of the system is defined by the expression $E_{\mathrm{B}}=E_{\text {free }}-E_{\text {total }}$, where the first term is the energy of the system in the absence of Coulomb interaction. The defined in such a way binding energy is positive.

\section{Results}

The binding energy $E_{\mathrm{B}}$ as a function of the external magnetic field $\boldsymbol{B} \| \boldsymbol{z}$ has been calculated for off-centre position of the donor in the $x y$ plane and for any 
position of donor centre along the $\mathrm{z}$ direction. We have obtained a nonmonotonic behaviour of $E_{\mathrm{B}}(B)$, presented in Fig. 3. One important parameter of the problem is the diameter of the quantum dot. It was taken from the experiment - as the middle value $R_{\mathrm{f}}$ from the range of $\mathrm{QD}$ diameters deduced on the base of the position of $A$ peak (see Fig. 2). The two remaining parameters: (a) position of donor centre in the quantum dot, (b) depth of the limiting potential in $x y$ plane $-\Delta V$, were determined from the calculations.
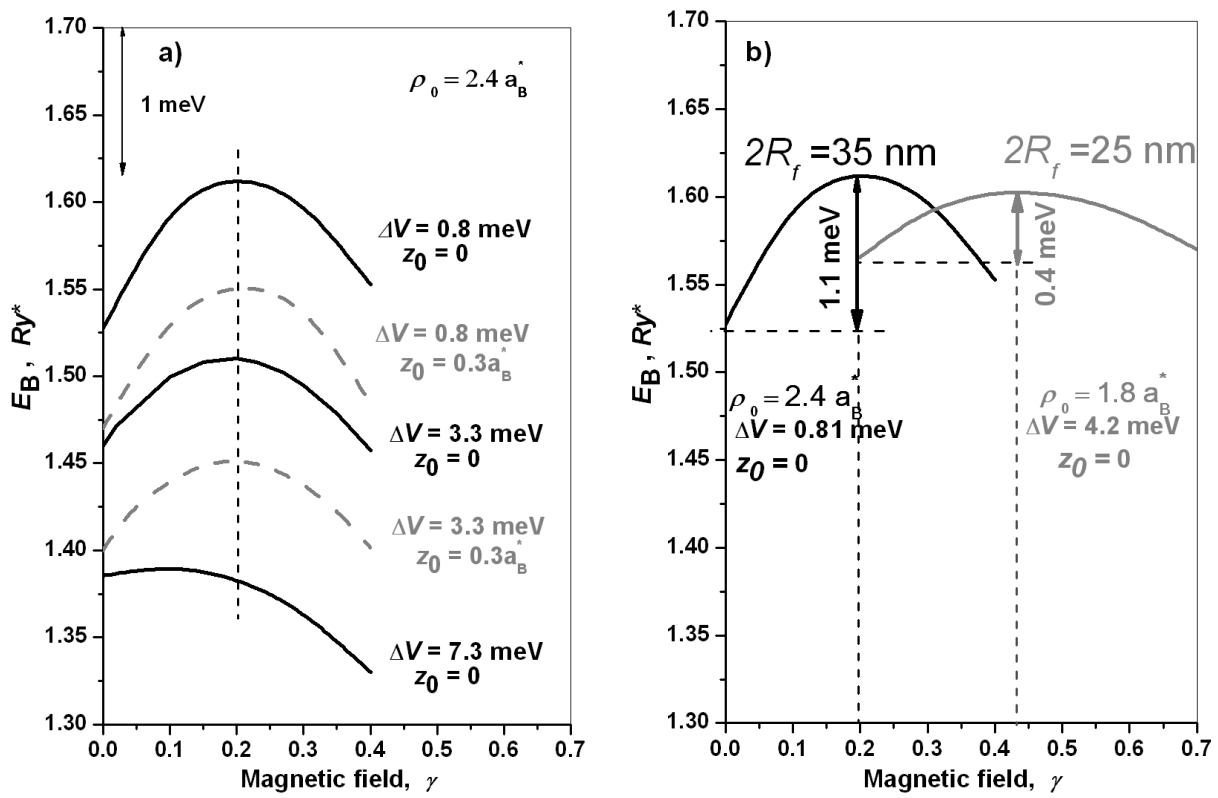

Fig. 3. (a) Binding energy (in units of $R y^{*}$ ) of the $1 s$ state of an electron on a donor as a function of the magnetic field in a CdTe quantum dot with the diameter $2 R_{\mathrm{f}}=35 \mathrm{~nm}$, for different values of the depth of the confining potential $\Delta V$ in the $x y$ plane and for different positions of the donor along the $z$ direction $\left(z_{0}=0\right.$ corresponds to the middle of the QD). The donor shift parameter in $x y$ plane is equal to $\rho_{0}=2.4 a_{\mathrm{B}}^{*}$. (b) Comparison of the $E_{\mathrm{B}}(\gamma)$ dependence for two dots of a different $x y$ spatial extent. The assumed (on the basis of the effective mass approximation) parameters for CdTe are: $R y^{*}=13 \mathrm{meV}$, $a_{\mathrm{B}}^{*}=5.6 \mathrm{~nm}$.

Figure 3a shows that the maximum of the curve describing a dependence of the binding energy of electron in the quantum dot as a function of the magnetic field occurs for the same value of the magnetic field $\gamma \approx 0.2$ for different values of $\Delta V$ and $\gamma_{\mathrm{p}}$ (where the relation: $\gamma_{\mathrm{p}}^{2}\left(R_{\mathrm{f}} / a_{\mathrm{B}}^{*}\right)^{2}=\Delta V / R y^{*}$ is fulfilled). For the largest value of $\Delta V$ (see the lowest curve in Fig. 3a, which corresponds to $\left.\gamma_{\mathrm{p}}=0.3\right), E_{\mathrm{B}}$ only decreases with increasing $\boldsymbol{B}$. Hence, we have to take into considerations lower values of $\Delta V$ which correspond to $\gamma_{\mathrm{p}}$ from the range $\sim(0.1-0.2)$. This gives the 
limit of the maximum potential depth (in the $x y$ plane) to be equal to $\sim 3 \mathrm{meV}$. This is in agreement with the experimental conclusion that the depth of larger fluctuation has to be significantly greater than $0.3 \mathrm{meV}$. The results obtained for different $z$ position of the donor in the QD (see the dashed lines in Fig. 3a) show that the change of donor position along the $z$ direction does change the value of the binding energy (which is in agreement with a general behaviour of donors in low dimensionality systems) but the maximum of binding energy remains at the same magnetic field. The comparison of the $E_{\mathrm{B}}(\gamma)$ dependence for two dots of different $x y$ spatial extent is presented in Fig. $3 \mathrm{~b}$. It is visible that the maxima of the two curves occur at different magnetic fields. Moreover, the binding energy of an electron is roughly the same. This means that we may observe localization in each dot at different $B$ for the same energy of intra donor transitions from the ground state $1 s$ to the excited state. We can prove the ground state position by measuring the $1 s-2 p$ transitions because the value of $2 p$ shallow donor excited state in the QW [2] almost does not depend on other factors except $B$. Calculating a difference between the maximum of the curve in Fig. $3 \mathrm{~b}$ and the value in low magnetic field we see that an increase in $B$ influences larger QD more significantly, giving the effect of a localization comparable with the potential of the depth equal to $\sim 1.1 \mathrm{meV}$. Smaller QD in Fig. 3b exhibit an effect of localization equivalent to the depth $\sim 0.4 \mathrm{meV}$. The latter value is close to the thermal energy at $T=4.2 \mathrm{~K}$. We see that the localization increases when the magnetic field grows, but it might be disturbed at higher temperatures, which corresponds to depopulation of donor states observed in an experiment. This explains the lack of peak $B$ at $4.2 \mathrm{~K}$.

\section{Conclusions}

The presented experimental results indicate that the presence of in-plane potential fluctuations in a uniformly doped quantum well. In our experiments we observe two peaks in the magnetophotoconductivity spectra which are interpreted as the evidence of the presence in the sample the fluctuations with a characteristic size of 23-25 nm and 30-40 nm. An analysis of the influence of temperature on the spectra leads to the conclusion that the localization in smaller fluctuations is weaker and in that sense the observed fluctuations are similar to random fluctuations considered in 3D systems [6]. The presented theoretical analysis is a good approximation of the situation in our system. It describes the behaviour of a single electron in a fluctuation which is modelled as a parabolic quantum dot. It explains very well the experimentally observed lack of dependence of shallow donor transitions on the FIR photon energy. It also explains the shallower character of smaller fluctuations in the sample.

\section{Acknowledgments}

This work was supported by Armenian State Program "Semiconductor Nanoelectronics" and NFSAT. 


\section{References}

[1] G. Bastard, Phys. Rev. B 24, 4714 (1981).

[2] R.L. Green, K.K. Bajaj, Phys. Rev. B 34, 951 (1986).

[3] S. Huant, M. Grynberg, G. Martinez, B. Etienne, B. Lambert, A. Regreny, Solid State Commun. 65, 1467 (1988).

[4] M. Szot, K. Karpierz, J. Kossut, M. Grynberg, Phys. Status Solidi C 2, 609 (2003).

[5] M. Szot, K. Karpierz, J. Kossut, M. Grynberg, AIP Conf. Proc. 772, 1129 (2005).

[6] B.I. Shklovskii, A.L. Efros, Electronic Properties of Doped Semiconductors, Springer-Verlag, Berlin 1984.

[7] A.A. Avetisyan, A.P. Djotyan, E.M. Kazaryan, Phys. Status Solidi C 2, 734 (2003). 